\title{
NOETHER-LEFSCHETZ LOCUS FOR SURFACES
}

\author{
SUNG-OCK KIM
}

\begin{abstract}
We generalize M. Green's Explicit Noether-Lefschetz Theorem to the family of smooth complete intersection surfaces in the higher dimensional projective spaces. Moreover, we give a new proof of the Density Theorem due to C. Ciliberto, J. Harris, and R. Miranda [5].
\end{abstract}

\section{INTRODUCTION}

Let $\mathbf{P}^{n}$ be the complex projective space of dimension $n$. The NoetherLefschetz Theorem says that a general surface $S$ of degree $d$ in $\mathbf{P}^{3}$ contains only curves which are complete intersections of $S$ with another hypersurface in $\mathbf{P}^{3}$ for $d \geq 4$. The word "general" is used in the following sense: A property is said to hold at a general point of a projective variety $V$, if there exists a countable union $\Sigma$ of proper subvarieties of $V$ such that the property holds at all points of $V-\Sigma$. In [21], Lefschetz proved an even more general version: A general complete intersection surface $S$ of $n-2$ hypersurfaces in $\mathbf{P}^{n}, n \geq 3$, contains only curves that are themselves complete intersections unless $S$ is an intersection of two quadric 3 -folds in $\mathbf{P}^{4}$ or degree $S \leq 3$ in $\mathbf{P}^{3}$. We denote by $Y_{n}$ the space of smooth complete intersection surfaces of type $\left(d_{1}, \ldots, d_{n-2}\right)$ in $\mathbf{P}^{n}$, where $2 \leq d_{1} \leq d_{2} \leq \cdots \leq d_{n-2}$. Let $E=\bigoplus_{i=1}^{n-2} \mathscr{O}_{\mathbf{P}^{n}}\left(d_{i}\right) . \quad Y_{n}$ is parametrized by an open subset, which is also denoted by $Y_{n}$, by abuse of notation, of the Grassmannian of 1-dimensional subspaces of $H^{0}\left(\mathbf{P}^{n}, E\right)$. The Noether-Lefschetz locus $\Sigma_{n}$ in $Y_{n}$ is the set of smooth surfaces in $Y_{n}$ containing curves which are not complete intersections, i.e.,

$$
\Sigma_{n}=\left\{S \in Y_{n} \mid \operatorname{Pic}(S) \text { is not generated by the hyperplane class }\right\} .
$$

Since the fundamental work of Noether and Lefschetz, their results have been improved in a number of interesting directions (see, e.g., $[3,7,11,12,23,26$, 27]). For $n=3$, by a mixture of Hodge-theoretic and algebraic techniques, Green $[8,10]$ showed that every irreducible component of $\Sigma_{3}$ has codimension at least $d_{1}-3$ in the family of smooth surfaces of degree $d_{1}$ in $\mathbf{P}^{3}$ for $d_{1} \geq 3$,

Received by the editors November 20,1989. The contents of this paper have been presented January 19, 1990.

1980 Mathematics Subject Classification (1985 Revision). Primary 14J10; Secondary 14B10.

This paper is part of the author's Ph. D. Thesis at the University of California, Los Angeles. The author wishes to express her special thanks to her thesis advisor Mark L. Green. 
which is called the explicit Noether-Lefschetz Theorem. A generalization of this theorem to the case $n \geq 4$ is given in $\S 2$ (cf. Theorem 1). There is one new phenomenon in this case which is not present in the case of surfaces in $\mathbf{P}^{3}$. For example, the analog of Green's result in $\mathbf{P}^{4}$ holds only when a general element of a component is the intersection of two smooth 3-folds.

On the other hand, an upper bound for the codimension of the irreducible components in the case $n=3$ is $p_{g}=\left(\begin{array}{c}d_{1}-1 \\ 3\end{array}\right)$. Ciliberto, Harris and Miranda [5] proved that over an algebraically closed field of any characteristic, for $d_{1} \geq 4$, the Noether-Lefschetz locus in the family $Y_{3}$ of smooth surfaces of degree $d_{1}$ in $\mathbf{P}^{3}$ contains infinitely many components having maximal codimension $p_{g}$ and the union of these components is Zariski dense in $Y_{3}$. Following $\mathrm{M}$. Green's idea, they showed that over the complex numbers, the existence of one such component implies that the union of the components having maximal codimension $p_{g}$ is dense in $Y_{3}$ in the classical topology. We will give a rather simple infinitesimal proof of this without constructing such components directly in $\S 3$.

\section{A generalization of the explicit Noether-Lefschetz Theorem}

Let $\Sigma_{L} \subset \Sigma_{n}$ denote the subvariety of surfaces containing lines, i.e., curves of degree 1 in $\mathbf{P}^{n}$. Let $G=$ Grassmannian of lines in $\mathbf{P}^{n}$. Then $\Sigma_{L}$ is the image under projection on the second factor of the incidence correspondence

$$
\widetilde{\Sigma}_{L}=\{(C, S) \mid C \subset S\} \subset G \times Y_{n} .
$$

For a line $l \subset \mathbf{P}^{n}$, we have an exact sequence

$$
0 \rightarrow \mathscr{J}_{l \mid \mathbf{P}^{n}} \rightarrow \mathscr{O}_{\mathbf{P}^{n}} \stackrel{r}{\longrightarrow} \mathscr{O}_{l} \rightarrow 0
$$

where $r$ is the restriction map. Tensoring with $E$ and taking the long exact sequence of cohomology, we have

$$
0 \rightarrow H^{0}\left(\mathbf{P}^{n}, \mathscr{I}_{l \mid \mathbf{P}^{n}} \otimes E\right) \rightarrow H^{0}\left(\mathbf{P}^{n}, E\right) \rightarrow H^{0}\left(\mathbf{P}^{n}, E \otimes \mathscr{O}_{l}\right) \rightarrow \cdots .
$$

From this sequence, we can see that the fiber of the projection map $\pi_{1}: \tilde{\Sigma}_{L} \rightarrow G$ over $l$ is contained in $H^{0}\left(\mathbf{P}^{n}, \mathscr{I}_{l \mid \mathbf{P}^{n}} \otimes E\right)$. Since $H^{0}\left(\mathbf{P}^{n}, E\right) \rightarrow H^{0}\left(\mathbf{P}^{n}, E \otimes \mathscr{O}_{l}\right)$ is surjective,

$$
\operatorname{dim} \text { fiber of } \pi_{1}=\operatorname{dim} H^{0}\left(\mathbf{P}^{n}, E\right)-\sum_{i=1}^{n-2}\left(d_{i}+1\right)-1 .
$$

So

$$
\operatorname{codim}_{Y_{n}} \Sigma_{L}=\sum_{i=1}^{n-2} d_{i}-n
$$

We have the following explicit Noether-Lefschetz Theorem for $n \geq 4$, which generalizes the theorem of Green [10] for $n=3$. 
Theorem 1. Let $n \geq 4$. An irreducible component $\Sigma^{\prime}$ of $\Sigma_{n}$ has codimension at least $\sum_{i=1}^{n-2} d_{i}-n$ in $Y_{n}$ iffor a general point of $\Sigma^{\prime}$, the corresponding surface $S=\bigcap_{i=1}^{n-2} H_{i}$ has the property that $\bigcap_{i=1}^{k} H_{i}$ has no singularity for any $k$ with $d_{k}<d_{k+1}$.

Example. The hypothesis in Theorem 1 is necessary. For example, let $n=4$ and $F_{1}=z_{0}^{d_{1}}+z_{0} z_{2}^{d_{1}-1}+z_{1}^{d_{1}}+z_{1} z_{3}^{d_{1}-1}$. Then $H_{1}=\left\{F_{1}=0\right\}$ has an isolated singularity at $(0,0,0,0,1)$ and has no other singularities. It is a cone over a smooth surface in $\mathbf{P}^{3}$ containing a line. $H_{1}$ contains the plane $z_{0}=z_{1}=0$. The intersection of $H_{1}$ with any 3-fold $H_{2}$ of degree $d_{2}$ contains a plane curve of degree $d_{2}$ and therefore is in the Noether-Lefschetz locus. The component $\Sigma^{\prime}$ containing these complete intersection surfaces has codimension depending only on $d_{1}$, i.e., codimension of $\Sigma^{\prime}$ is at most $\left(\begin{array}{c}d_{1}+4 \\ 4\end{array}\right)$. If $d_{2}>\left(\begin{array}{c}d_{1}+4 \\ 4\end{array}\right)+4-d_{1}$, then $\operatorname{codim}_{Y_{4}} \Sigma^{\prime}<d_{1}+d_{2}-4$.

We will give a proof of Theorem 1 using similar techniques to Green's [10]. First, we will show the following simple algebraic fact and then reduce our theorem to this.

Proposition 1. Let $W \subseteq H^{0}\left(\mathbf{P}^{n}, E\right)$ be a subspace such that the evaluation map

$$
W \otimes \mathscr{O}_{\mathbf{P}^{n}, x} \rightarrow E_{x}
$$

is surjective for all $x \in \mathbf{P}^{n}$. Then the map

$$
W \otimes H^{0}\left(\mathbf{P}^{n}, \mathscr{O}_{\mathbf{P}^{n}}(k)\right) \rightarrow H^{0}\left(\mathbf{P}^{n}, E(k)\right)
$$

is surjective if $k \geq \operatorname{codim} W$.

Proof. Let $c=\operatorname{codim} W$. We can choose an increasing sequence of linear subspaces

$$
W_{c}=W \subset W_{c-1} \subset \cdots \subset W_{1} \subset W_{0}=H^{0}\left(\mathbf{P}^{n}, E\right)
$$

so that $\operatorname{dim} W_{i-1} / W_{i}=1$ for $i=1,2, \ldots, c$. Since the evaluation map $W \otimes$ $\mathscr{O}_{\mathbf{P}^{n}, x} \rightarrow E_{x}$ is surjective at all $x \in \mathbf{P}^{n}$, the kernel $M_{i}$ of the map $W_{i} \otimes \mathscr{O}_{\mathbf{P}^{n}} \rightarrow E$ is a vector bundle on $\mathbf{P}^{n}$ sitting in the exact sequence

$$
0 \rightarrow M_{i} \rightarrow W_{i} \otimes \mathscr{O}_{\mathbf{P}^{n}} \rightarrow E \rightarrow 0
$$

for $i=1,2, \ldots, c$, and it is enough to show that

$$
H^{1}\left(M_{c} \otimes \mathscr{O}_{\mathbf{P}^{n}}(k)\right)=0 \quad \text { if } k \geq c=\operatorname{codim} W,
$$

which follows from the following lemma.

Lemma. For all $i=0,1, \ldots, c, H^{q}\left(\mathbf{P}^{n}, \bigwedge^{p} M_{i}(k)\right)=0$ if $q \geq 1$ and $k+q \geq$ $p+i$.

Proof. We note that the $M_{i}$ 's sit in the exact sequence

$$
0 \rightarrow M_{i} \rightarrow M_{i-1} \rightarrow \mathscr{O}_{\mathbf{P}^{n}} \rightarrow 0
$$


and thus we have an exact sequence

$$
0 \rightarrow \bigwedge^{p+1} M_{i} \rightarrow \bigwedge^{p+1} M_{i-1} \rightarrow \bigwedge^{p} M_{i} \rightarrow 0
$$

for each $i$. Tensoring by $\mathscr{O}_{\mathbf{P}^{n}}(k)$ and taking the long exact sequence on cohomology, we have

$$
\begin{aligned}
\cdots & \rightarrow H^{q}\left(\mathbf{P}^{n}, \bigwedge^{p+1} M_{i-1}(k)\right) \rightarrow H^{q}\left(\mathbf{P}^{n}, \bigwedge^{p} M_{i}(k)\right) \\
& \rightarrow H^{q+1}\left(\mathbf{P}^{n}, \bigwedge^{p+1} M_{i}(k)\right) \rightarrow \cdots .
\end{aligned}
$$

Let $q \geq 1$ and $k+q \geq p+i, i=0,1, \ldots, c$. We will use induction on $i$ and $p$ to prove the lemma. First, notice that if $p \geq \operatorname{rank} M_{i}$, then $H^{q}\left(\mathbf{P}^{n}, \wedge^{p+1} M_{i}(k)\right)=0$ for all $q \geq 0$ and for any $k \geq 0$.

Sublemma. For $i=0, H^{q}\left(\mathbf{P}^{n}, \bigwedge^{p} M_{0}(k)\right)=0$ if $q \geq 1$ and $k+q \geq p$.

To see this, we first recall (cf. [24, Lecture 14]) that a coherent sheaf $F$ on $\mathbf{P}^{n}$ is said to be $m$-regular, if $H^{q}\left(\mathbf{P}^{n}, F(m-q)\right)=0$ for $q>0$.

From the exact sequence

$$
0 \rightarrow M_{0} \rightarrow H^{0}\left(\mathbf{P}^{n}, E\right) \otimes \mathscr{O}_{\mathbf{P}^{n}} \rightarrow E \rightarrow 0
$$

tensored with $\mathscr{O}_{\mathbf{P}^{n}}(1-q)$, we have the long exact sequence on the cohomology

$$
\begin{aligned}
\cdots & \rightarrow H^{q-1}\left(\mathbf{P}^{n}, E(1-q)\right) \rightarrow H^{q}\left(\mathbf{P}^{n}, M_{0}(1-q)\right) \\
& \rightarrow H^{0}\left(\mathbf{P}^{n}, E\right) \otimes H^{q}\left(\mathbf{P}^{n}, \mathscr{O}_{\mathbf{P}^{n}}(1-q)\right) \rightarrow \cdots .
\end{aligned}
$$

If $q=1$, then $H^{0}\left(\mathbf{P}^{n}, E\right) \otimes H^{0}\left(\mathbf{P}^{n}, \mathscr{O}_{\mathbf{P}^{n}}\right) \rightarrow H^{0}\left(\mathbf{P}^{n}, E\right)$ is an isomorphism and $H^{1}\left(\mathbf{P}^{n}, \mathscr{O}_{\mathbf{P}^{n}}\right)=0$, hence $H^{1}\left(\mathbf{P}^{n}, M_{0}\right)=0$.

For $q>1, H^{q-1}\left(\mathbf{P}^{n}, E(1-q)\right)=0$ and $H^{q}\left(\mathbf{P}^{n}, \mathscr{O}_{\mathbf{P}^{n}}(1-q)\right)=0$, and hence

$$
H^{q}\left(\mathbf{P}^{n}, M_{0}(1-q)\right)=0 .
$$

Thus $M_{0}$ is 1-regular. Then $\bigwedge^{p} M_{0}$ is $p$-regular (see, e.g., [20, Lemma 2.7]). Since $p$-regularity implies $(p+1)$-regularity [24, loc.cit], the sublemma follows.

By ascending induction on $i$, we may assume

$$
H^{q}\left(\mathbf{P}^{n}, \bigwedge^{p+1} M_{i-1}(k)\right)=0
$$

since $k+q \geq(p+1)+(i-1)=p+i$. By descending induction on $p$, we may assume

$$
H^{q+1}\left(\mathbf{P}^{n}, \bigwedge^{p+1} M_{i}(k)\right)=0
$$

since $k+q \geq p+i$ which is equivalent to $k+(q+1) \geq(p+1)+i$. Hence

$$
H^{q}\left(\mathbf{P}^{n}, \bigwedge^{p} M_{i}(k)\right)=0 \text {, }
$$

and the lemma follows. 
For a compact complex manifold $M$ of dimension $n$ with the associated $(1,1)$-form $\omega$, we recall that the primitive cohomology is

$$
H_{\mathrm{pr}}^{n-k}(M)=\operatorname{ker}\left\{\omega^{k+1}: H^{n-k}(M) \rightarrow H^{n+k+2}(M)\right\} .
$$

We denote $H_{\mathrm{pr}}^{p, q}(M)=H^{p, q}(M) \cap H_{\mathrm{pr}}^{p+q}(M)$. For a smooth hypersurface $X$ of degree $d$ in $\mathbf{P}^{n}$ with defining equation $F\left(z_{0}, \ldots, z_{n}\right)=0$, it is known (cf. $[4,15])$ that there are natural Poincaré residue isomorphisms

$$
H_{\mathrm{pr}}^{n-\dot{k}-1, k}(X) \simeq S^{d(k+1)-n-1} / J_{F, d(k+1)-n-1}
$$

where $S=\bigoplus_{k \geq 0} S^{k}$ is the graded ring $\mathbf{C}\left[z_{0}, \ldots, z_{n}\right]$ and $J_{F}=\bigoplus_{k \geq d-1} J_{F, k}$ denotes the Jacobian ideal of $F$ generated by the first partial derivatives of $F$. In the proof of Theorem 1, we will use this kind of algebraic representations of $H^{2,0}(S)$ and $H_{\mathrm{pr}}^{1,1}(S)$ for $S \in Y_{n}$.

We need the following special cases of the Bott Vanishing Theorem (cf. [2]):

Bott Vanishing Theorem. $H^{p}\left(\mathbf{P}^{n}, \Omega_{\mathbf{P}^{n}}^{q}(k)\right)=0$ unless

(i) $p=q$ and $k=0$,

(ii) $p=0$ and $k>q$, or

(iii) $p=n$ and $k<q-n$.

We will also use the following well-known fact (see, e.g., [18, pp. 445-446]): (2.2) Let

$$
0 \rightarrow \mathscr{K}^{0} \rightarrow \cdots \rightarrow \mathscr{K}^{m} \rightarrow 0
$$

be an exact sequence of sheaves on a topological space $X$. Then there is a spectral sequence abutting to zero with $E_{1}^{p, q}=H^{q}\left(X, \mathscr{K}^{p}\right)$.

Let $B=\bigoplus_{i=1}^{n-2} \mathscr{O}_{\mathbf{P}^{n}}\left(-d_{i}\right)$. Then for $S \in Y_{n}$, there is a Koszul complex

$$
0 \rightarrow \bigwedge^{n-2} B \rightarrow \bigwedge^{n-3} B \rightarrow \cdots \rightarrow \bigwedge^{2} B \rightarrow B \rightarrow \mathscr{O}_{\mathbf{P}^{n}} \rightarrow \mathscr{O}_{S} \rightarrow 0
$$

which is exact since $S$ is a complete intersection (see, e.g., [18, p. 688]). We denote $\mu=\sum_{i=1}^{n-2} d_{i}-n-1$. For an algebraic representation of $H^{2,0}(S)$, tensoring (2.3) with $\mathscr{O}_{\mathbf{P}^{n}}(\mu)$ and applying (2.2), we obtain a spectral sequence abutting to zero with $E_{1}^{p, q}=0$ unless $q=0, q=n$, or $p=n-1$. There is no nonzero differential other than the differentials in $E_{1}$ coming into the position $(p, 0)$ for $p=0,1, \ldots, n-1$. So we obtain an exact sequence

$$
\cdots \rightarrow H^{0}\left(\mathbf{P}^{n}, \bigoplus_{i=1}^{n-2} \mathscr{O}_{\mathbf{P}^{n}}\left(\mu-d_{i}\right)\right) \rightarrow H^{0}\left(\mathbf{P}^{n}, \mathscr{O}_{\mathbf{P}^{n}}(\mu)\right) \rightarrow H^{0}\left(\mathbf{P}^{n}, \mathscr{O}_{S}(\mu)\right) \rightarrow 0
$$

and hence

$$
H^{2,0}(S) \simeq H^{0}\left(\mathbf{P}^{n}, \mathscr{O}_{\mathbf{P}^{n}}(\mu)\right) / \operatorname{im} H^{0}\left(\mathbf{P}^{n}, \bigoplus_{i=1}^{n-2} \mathscr{O}_{\mathbf{P}^{n}}\left(\mu-d_{i}\right)\right)
$$


For an algebraic representation of $H_{\mathrm{pr}}^{1,1}(S)$ for $S \in Y_{n}$, we take the long exact sequence on the cohomology of the short exact sequence

$$
\left.0 \rightarrow \Theta_{S} \otimes K_{S} \rightarrow \Theta_{\mathbf{P}^{n}}\right|_{S} \otimes K_{S} \rightarrow N_{S \mid \mathbf{P}^{n}} \otimes K_{S} \rightarrow 0,
$$

where $\Theta_{S}$ and $N_{S \mid \mathbf{P}^{n}}$ denote the holomorphic tangent bundle of $S$ and the normal bundle of $S$ in $\mathbf{P}^{n}$, respectively. Then we get

$$
\begin{aligned}
& \rightarrow H^{0}\left(S,\left.\Theta_{\mathbf{P}^{n}}\right|_{S} \otimes K_{S}\right) \rightarrow H^{0}\left(S, N_{S \mid \mathbf{P}^{n}} \otimes K_{S}\right) \rightarrow H^{1}\left(S, \Theta_{S} \otimes K_{S}\right) \\
& \rightarrow H^{1}\left(S,\left.\Theta_{\mathbf{P}^{n}}\right|_{S} \otimes K_{S}\right) \rightarrow \cdots
\end{aligned}
$$

So

$$
\frac{H^{0}\left(S, N_{S \mid \mathbf{P}^{n}} \otimes K_{S}\right)}{\operatorname{im} H^{0}\left(S,\left.\Theta_{\mathbf{P}^{n}}\right|_{S} \otimes K_{S}\right)} \simeq\left(\frac{H^{1}\left(S, \Omega_{S}^{1}\right)}{\operatorname{im} H^{1}\left(S,\left.\Omega_{\mathbf{P}^{n}}\right|_{S}\right)}\right)^{*}
$$

by Serre duality. We will show that

$$
H_{\mathrm{pr}}^{1,1}(S) \simeq \frac{H^{1}\left(S, \Omega_{S}^{1}\right)}{\operatorname{im} H^{1}\left(S, \Omega_{\left.\mathbf{P}^{n}\right|_{S}}^{1}\right)} .
$$

Applying (2.2) to the exact sequence (2.3) tensored with $\Omega_{\mathrm{P}^{n}}^{1}$, we get a spectral sequence abutting to zero. By the Bott Vanishing Theorem, $E_{1}^{p, q}=0$ unless $q=0, n$, or $p=n-1$, or $(p, q)=(n-2,1)$. Moreover, no nonzero differential except the differential in $E_{1}$ comes into or goes out of the position $(n-2,1)$ or $(n-1,1)$. So $H^{1}\left(\mathbf{P}^{n}, \Omega_{\mathbf{P}^{n}}^{1}\right)=H^{1}\left(S, \Omega_{\left.\mathbf{P}^{n}\right|_{S}}^{1}\right)$. From the exact sequence (2.3) tensored with the dual $E^{*}$ of $E$, we get a spectral sequence abutting to zero with $E_{1}^{p, q}=0$ unless $q=0$, or $q=n$, or $p=n-1$. No nonzero differential comes into the position $(n-1,1)$. So $H^{1}\left(S, \mathscr{O}_{S} \otimes E^{*}\right)=0$. We note that $N_{S \mid \mathbf{P}^{n}}=\mathscr{O}_{S} \otimes E$. Thus

$$
\operatorname{im} H^{1}\left(S, \Omega_{\left.\mathbf{P}^{n}\right|_{S}}^{1}\right) \simeq H^{1}\left(S, \Omega_{\left.\mathbf{P}^{n}\right|_{S}}^{1}\right) \simeq H^{1}\left(\mathbf{P}^{n}, \Omega_{\mathbf{P}^{n}}^{1}\right) \simeq(\omega),
$$

where $\omega$ is the associated $(1,1)$ form of $\mathbf{P}^{n}$ (i.e., $\omega$ is the first Chern class $c_{1}\left(\mathscr{O}_{\mathbf{P}^{n}}(1)\right)$ of $\left.\mathscr{O}_{\mathbf{P}^{n}}(1)\right)$. By Lefschetz decomposition, $\left.H^{1}\left(\Omega_{S}^{1}\right) \simeq H_{\mathrm{pr}}^{1,1}(S) \oplus \omega\right|_{S}$. $H^{0,0}(S)$. Hence we get (2.4). From the spectral sequence attached to the exact sequence (2.3) tensored with $E(\mu)$, we can see that

$$
0 \rightarrow H^{0}\left(\mathbf{P}^{n}, E(-n-1)\right) \rightarrow \cdots \rightarrow H^{0}\left(\mathbf{P}^{n}, E(\mu)\right) \stackrel{r}{\longrightarrow} H^{0}\left(S, N_{S \mid \mathbf{P}^{n}} \otimes K_{S}\right) \rightarrow 0
$$

is exact. Hence

$$
\frac{H^{0}\left(S, N_{S \mid \mathbf{P}^{n}} \otimes K_{S}\right)}{\operatorname{im} H^{0}\left(S,\left.\Theta_{\mathbf{P}^{n}}\right|_{S} \otimes K_{S}\right)} \simeq \frac{H^{0}\left(\mathbf{P}^{n}, E(\mu)\right)}{r^{-1}\left(\operatorname{im} H^{0}\left(\left.\Theta_{\mathbf{P}^{n}}\right|_{S} \otimes K_{S}\right)\right)} .
$$

Summarizing the above computations, we obtain the following identifications: 


\section{Proposition 2.}

$$
\begin{aligned}
H^{2,0}(S) & \simeq \frac{H^{0}\left(\mathbf{P}^{n}, \mathscr{O}_{\mathbf{P}^{n}}(\mu)\right)}{\operatorname{im} H^{0}\left(\mathbf{P}^{n}, \bigoplus_{i=1}^{n-2} \mathscr{O}_{\mathbf{P}^{n}}\left(\mu-d_{i}\right)\right)} \\
H_{\mathrm{pr}}^{1,1}(S)^{*} & \simeq \frac{H^{0}\left(S, N_{S \mid \mathbf{P}^{n}} \otimes K_{S}\right)}{\operatorname{im} H^{0}\left(S,\left.\Theta_{\mathbf{P}^{n}}\right|_{S} \otimes K_{S}\right)} \simeq \frac{H^{0}\left(\mathbf{P}^{n}, E \otimes \mathscr{O}_{\mathbf{p}^{n}}(\mu)\right)}{r^{-1}\left(\operatorname{im} H^{0}\left(S,\left.\Theta_{\mathbf{P}^{n}}\right|_{S} \otimes K_{S}\right)\right)}
\end{aligned}
$$

We also need an algebraic representation of the subspace of $H^{1}\left(S, \Theta_{S}\right)$ parametrizing the deformations of $S$ in $\mathbf{P}^{n}$, that is, the image of the Zariski tangent space $T_{S}\left(Y_{n}\right)$ of $Y_{n}$ at $S$ under the Kodaira-Spencer map $\rho: T_{S}\left(Y_{n}\right) \rightarrow$ $H^{1}\left(S, \Theta_{S}\right)$. Let $S=\bigcap_{i=1}^{n-2}\left\{F_{i}=0\right\} . T_{S}\left(Y_{n}\right)$ is naturally isomorphic to

$$
\operatorname{Hom}\left((S), H^{0}\left(\mathbf{P}^{n}, E\right) /(S)\right) \simeq H^{0}\left(\mathbf{P}^{n}, E\right) /(S),
$$

where $(S)$ denotes the 1-dimensional subspace of $H^{0}\left(\mathbf{P}^{n}, E\right)$ generated by $\left(F_{1}, \ldots, F_{n-2}\right)$. So the map $T_{S}(Y) \rightarrow H^{0}\left(S, N_{S \mid \mathbf{P}^{n}}\right)$ is surjective and

$$
\rho\left(T_{S}\left(Y_{n}\right)\right)=\operatorname{im}\left\{H^{0}\left(S, N_{S \mid \mathbf{P}^{n}}\right) \rightarrow H^{1}\left(S, \Theta_{S}\right)\right\} .
$$

Tensoring the exact sequence (2.3) with $\Theta_{\mathbf{P}^{n}}$ and applying (2.2), we have a spectral sequence abutting to zero. By Serre duality and the Bott Vanishing Theorem, $H^{0}\left(\mathbf{P}^{n}, \Theta_{\mathbf{P}^{n}}(k)\right)$ vanishes unless $-k-n-1<1-n$. Hence $E_{1}^{p, q}=0$ unless $(p, q)=(n-2,0),(n-1,0),(n-2,1),(n-1,1)$, or $q=n$. No nonzero differential except the differentials in $E_{1}$ comes into the position $(n-2,0)$ or $(n-1,0)$. Hence the map $\gamma_{1}: H^{0}\left(\mathbf{P}^{n}, \Theta_{\mathbf{P}^{n}}\right) \rightarrow H^{0}\left(S,\left.\Theta_{\mathbf{P}^{n}}\right|_{S}\right)$ is an isomorphism. From the spectral sequence attached to the exact sequence (2.3) tensored with $E$, we can see that the map $\gamma_{2}: H^{0}\left(\mathbf{P}^{n}, E\right) \rightarrow H^{0}\left(S, E \otimes \mathscr{O}_{S}\right)$ is surjective. From the short exact sequence

$$
\left.0 \rightarrow \Theta_{S} \rightarrow \Theta_{\mathbf{P}^{n}}\right|_{S} \rightarrow N_{S \mid \mathbf{P}^{n}} \rightarrow 0,
$$

we get the following long exact sequence which fits into a diagram:

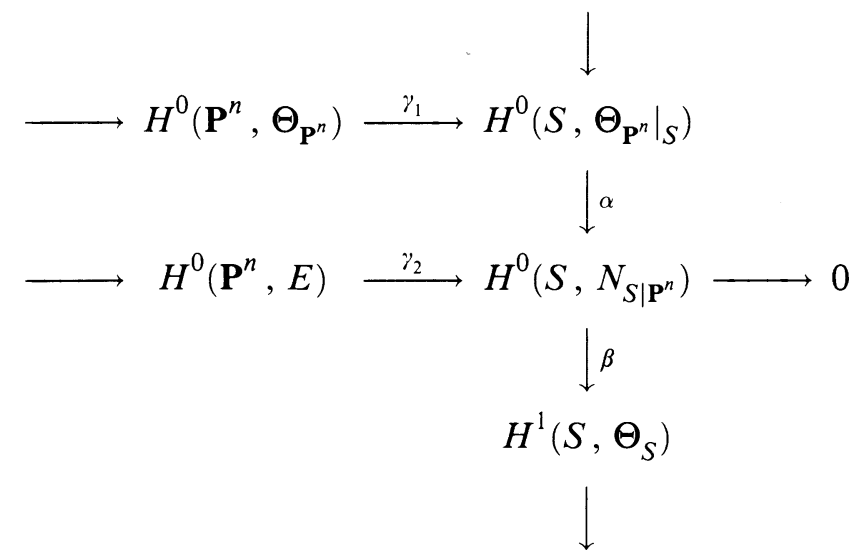


Hence

$$
\rho\left(T_{S}\left(Y_{n}\right)\right) \simeq \frac{H^{0}\left(S, N_{S \mid \mathbf{P}^{n}}\right)}{\alpha \circ \gamma_{1}\left(H^{0}\left(\mathbf{P}^{n}, \Theta_{\mathbf{P}^{n}}\right)\right)} \simeq \frac{H^{0}\left(\mathbf{P}^{n}, E\right)}{\gamma_{2}^{-1}\left(\alpha\left(H^{0}\left(S,\left.\Theta_{\mathbf{P}^{n}}\right|_{S}\right)\right)\right)} .
$$

Another preliminary fact we will use is the description of the Zariski tangent space to

$$
\widetilde{Y}_{n}=\left\{(S, L) \mid S \in Y_{n}, L \in \operatorname{Pic}(S)\right\} .
$$

The first prolongation bundle $P_{1}(L)$ of $L$ is defined by an exact sequence

$$
0 \rightarrow \Omega_{S}^{1} \otimes L \rightarrow P_{1}(L) \rightarrow L \rightarrow 0
$$

with the extension class $c_{1}(L) \in \operatorname{Ext}^{1}\left(L, \Omega_{S}^{1} \otimes L\right)=H^{1}\left(S, \Omega_{S}^{1}\right)$. The computation of Zariski tangent space to the set of pairs of curves with line bundles is given in [1]. An analogous argument gives the description for the surface case: For a fixed $(S, L)$, the Zariski tangent space $T_{(S, L)}\left(\widetilde{Y}_{n}\right)$ of $Y_{n}$ at $(S, L)$ maps into $H^{1}\left(S, P_{1}(L)^{*} \otimes L\right)$ as follows. As a complex manifold, the line bundle $L \rightarrow S$ is given by the data

$$
\left\{U_{\alpha}, z_{\alpha}, f_{\alpha \beta}, g_{\alpha \beta}\right\},
$$

where $\left\{U_{\alpha}\right\}$ is a finite open covering of $S, z_{\alpha}=\left(z_{\alpha_{1}}, z_{\alpha_{2}}\right)$ are local coordinates in $U_{\alpha}, f_{\alpha \beta}$ is the coordinate transformation on $U_{\alpha} \cap U_{\beta}$, and $g_{\alpha \beta}$ is the transition function for $L$. Thus two cocycle rules $f_{\alpha \gamma}=f_{\alpha \beta} \circ f_{\beta \gamma}$ and $g_{\alpha \gamma}=$ $g_{\alpha \beta} g_{\beta \gamma}$ hold in $U_{\alpha} \cap U_{\beta} \cap U_{\gamma}$. The first order deformation of $L \rightarrow S$ is given by

$$
\left\{U_{\alpha}, z_{\alpha}, f_{\alpha \beta}\left(z_{\beta}, t\right), g_{\alpha \beta}\left(z_{\beta}, t\right)\right\}
$$

satisfying

$$
\begin{gathered}
f_{\alpha \gamma}\left(z_{\gamma}, t\right) \equiv f_{\alpha \beta}\left(f_{\beta \gamma}\left(z_{\gamma}, t\right), t\right) \bmod t^{2} \\
g_{\alpha \gamma}\left(z_{\gamma}, t\right) \equiv g_{\alpha \beta}\left(f_{\beta \gamma}\left(z_{\gamma}, t\right), t\right) \cdot g_{\beta \gamma}\left(z_{\gamma}, t\right) \bmod t^{2}
\end{gathered}
$$

on $U_{\alpha} \cap U_{\beta} \cap U_{\gamma}$. Taking derivatives at $t=0$, we can see that $\tilde{f}_{\alpha \beta}=\left\{\frac{\partial f_{\alpha \beta}}{\partial t} \frac{\partial}{\partial z_{\alpha}}\right\}$ is a cocycle defining a class $\tilde{f}=\left\{\tilde{f}_{\alpha \beta}\right\}$ in $H^{1}\left(S, \Theta_{S}\right)$ and that $\left\{\tilde{g}_{\alpha \beta}=\frac{\partial g_{\alpha \beta}}{\partial t} g_{\alpha \beta}^{-1}\right\}$ is a 1-cochain with coefficients in $\mathscr{O}_{S}$. For the coboundary map $\delta, \delta\left(\left\{\tilde{g}_{\alpha \beta}\right\}\right)$ is the cup product of $\tilde{f}$ with $c_{1}(L)$. Note that $c_{1}(L)=\left\{g_{\alpha \beta}^{-1} d g_{\alpha \beta}\right\} \in H^{1}\left(S, \Omega_{S}^{1}\right)$. $\sigma=\left\{\left(\tilde{f}_{\alpha \beta}, \tilde{g}_{\alpha \beta}\right)\right\}$ defines a 1-cocycle with coefficients in the extension $M$ of $\Theta_{S}$ by $\mathscr{O}_{S}$, i.e., $M$ is defined by the exact sequence

$$
0 \rightarrow \mathscr{O}_{S} \rightarrow M \rightarrow \Theta_{S} \rightarrow 0
$$

with the extension class $c_{1}(L)$. But $M=P_{1}(L)^{*} \otimes L$. So

$$
(\sigma) \in H^{1}\left(S, P_{1}(L)^{*} \otimes L\right) .
$$


Proof of Theorem 1. Let $\widetilde{\Sigma}_{n}=\left\{(S, L) \mid S \in \Sigma_{n}\right.$ and $\left.L \in \operatorname{Pic}(S)\right\}$, and let $\pi:(S, L) \mapsto S$ be a projection. For $(S, L) \in \widetilde{Y}_{n}$, we have a commutative diagram

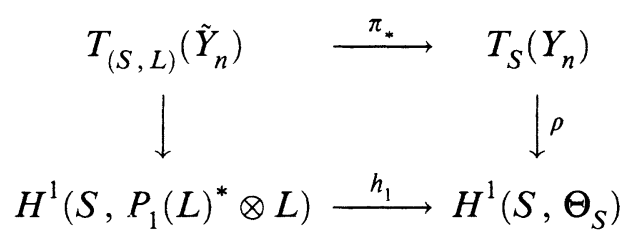

where $h_{1}$ sits in the long exact sequence on cohomology

$$
\rightarrow H^{1}\left(S, P_{1}(L)^{*} \otimes L\right) \stackrel{h_{1}}{\longrightarrow} H^{1}\left(S, \Theta_{S}\right) \stackrel{h_{2}}{\longrightarrow} H^{2}\left(S, \mathscr{O}_{S}\right) \rightarrow \cdots .
$$

Fix $(S, L) \in \widetilde{\Sigma}_{n}$ with $c_{1}(L) \in H_{\mathrm{pr}}^{1,1}(S)$. Let $Z$ be the union of all irreducible components of $\widetilde{\Sigma}_{n}$ containing $(S, L)$. The image $T(Z)$ of the Zariski tangent space $T_{S}(\pi(Z))$ of $\pi(Z)$ at $S$ under $\rho$ is in the kernel of $h_{2}$, i.e.,

$$
\begin{gathered}
\rho\left(T_{S}\left(Y_{n}\right)\right) \otimes H_{\mathrm{pr}}^{1,1}(S) \stackrel{\cup}{\longrightarrow} H^{2}\left(S, \mathscr{O}_{S}\right), \\
T(Z) \otimes H^{0}\left(S, K_{S}\right) \mapsto 0 .
\end{gathered}
$$

Equivalently,

$$
\begin{gathered}
\rho\left(T_{S}\left(Y_{n}\right)\right) \otimes H^{0}\left(S, K_{S}\right) \stackrel{\cup}{\longrightarrow} H_{\mathrm{pr}}^{1,1}(S)^{*}, \\
T(Z) \otimes H^{0}\left(S, K_{S}\right) \mapsto c_{1}(L)^{\perp} .
\end{gathered}
$$

Using the notations in the diagram (2.5), we set $T^{\prime}=\gamma_{2}^{-1} \circ \beta^{-1}(T(Z))$ $\subset H^{0}\left(\mathbf{P}^{n}, E\right)$. Then $T^{\prime} \supset \gamma_{2}^{-1}(\operatorname{im} \alpha) \supset \operatorname{ker} \gamma_{2}$ and the following holds:

Claim. If $S=\bigcap_{i=1}^{n-2}\left\{F_{i}=0\right\}$, and $F_{i}$ is a homogeneous polynomial of degree $d_{i}$ such that $\bigcap_{i=1}^{k}\left\{F_{i}=0\right\}$ is nonsingular for each $k$ with $d_{k}<d_{k+1}$, then the evaluation map $T^{\prime} \otimes \mathscr{O}_{\mathbf{P}^{n}, x} \rightarrow E_{x}$ is surjective at every $x \in \mathbf{P}^{n}$.

To see this, let $e_{i}=(0, \ldots, 0,1,0, \ldots, 0) \in \mathbf{C}^{n-2}$ denote the $i$ th coordinate vector, for $i=1, \ldots, n-2$. Then

$$
\begin{aligned}
\operatorname{ker} \gamma_{2} \supseteq\left\{F_{i} G_{k} e_{k} \mid G_{k} \in\right. & H^{0}\left(\mathbf{P}^{n}, \mathscr{O}_{\mathbf{P}^{n}}\left(d_{k}-d_{i}\right)\right), \\
& \left.d_{k} \geq d_{i}, \text { and } i=1, \ldots, n-2\right\} .
\end{aligned}
$$

We note that

$$
\gamma_{2}^{-1}(\operatorname{im} \alpha) \supseteq\left\{z_{l}\left(\frac{\partial F_{1}}{\partial z_{j}}, \ldots, \frac{\partial F_{n-2}}{\partial z_{j}}\right) \mid l, j=0,1, \ldots, n\right\} .
$$

For a fixed $x \in \mathbf{P}^{n}$, let $i_{0}$ denote the smallest number such that $x \in\left\{F_{i_{0}} \neq 0\right\}$. Then (i) $i_{0}=1$, or (ii) $i_{0}>1$ and $d_{i_{0}-1}<d_{i_{0}}$, or (iii) $i_{0}>1$ and $d_{i_{0}-1}=d_{i_{0}}$. We will show that the evaluation map at $x$ is surjective in any case.

Case (i): If $i_{0}=1$, then we can choose $G_{k} \in H^{0}\left(\mathbf{P}^{n}, \mathscr{O}_{\mathbf{P}^{n}}\left(d_{k}-d_{1}\right)\right), k=$ $1, \ldots, n-2$ so that $G_{k}(x) \neq 0$ for each $k$. So $\left\{F_{1}(x) G_{k}(x) e_{k} \mid k=1, \ldots, n-\right.$ 2) are $n-2$ linearly independent elements in $E_{x}$. 
Case (ii): If $i_{0}>1$ and $d_{i_{0}-1}<d_{i_{0}}$, then by the hypothesis of the claim $\bigcap_{i=1}^{i_{0}-1}\left\{F_{i}=0\right\}$ has no singularity and so there is a nonvanishing $\left(i_{0}-1\right) \times\left(i_{0}-1\right)$ minor of a matrix

say

$$
\left(\left.\frac{\partial F_{i}}{\partial z_{j}}\right|_{x}\right)_{\substack{i=1, \ldots, i_{0}-1 \\ j=0,1, \ldots, n}},
$$

$$
\left(\left.\frac{\partial F_{i}}{\partial z_{j}}\right|_{x}\right)_{\substack{i=1, \ldots, i_{0}-1 \\ j=j_{1}, \ldots, j_{i_{0}-1}}},
$$

which has rank $i_{0}-1$. Moreover, there is some $m$ such that $z_{m}(x) \neq 0$. We can choose $G_{k} \in H^{0}\left(\mathbf{P}^{n}, \mathscr{O}_{\mathbf{P}^{n}}\left(d_{k}-d_{i_{0}}\right)\right)$ for $k=i_{0}, i_{0}+1, \ldots, n-2$ so that $G_{k}(x) \neq 0$ for each $k$. Then

$$
\begin{gathered}
\left\{z_{m}\left(\frac{\partial F_{1}}{\partial z_{j}}, \ldots, \frac{\partial F_{n-2}}{\partial z_{j}}\right) \mid j=j_{1}, \ldots, j_{i_{0}-1}\right\} \\
\cup\left\{F_{i_{0}} G_{k} e_{k} \mid k=i_{0}, i_{0}+1, \ldots, n-2\right\}
\end{gathered}
$$

provides $n-2$ linearly independent elements in $E_{x}$ when evaluated at $x$.

Case (iii): If $i_{0}>1$ and $d_{i_{0}-1}=d_{i_{0}}$, let $i_{1}$ be the smallest number such that $d_{i_{1}}=\cdots=d_{i_{0}-1}=d_{i_{0}}$. Then $d_{i_{1}-1}<d_{i_{1}}$ and by the hypothesis of the claim, $\bigcap_{i=1}^{i_{1}-1}\left\{F_{i}=0\right\}$ has no singularity. So, as in (ii) we can find $j_{1}, \ldots, j_{i_{1}-1}$ such that

$$
\left(\left.\frac{\partial F_{i}}{\partial z_{j}}\right|_{x}\right)_{\substack{i=1, \ldots, i_{1}-1 \\ j=j_{1}, \ldots, j_{i_{1}}-1}}
$$

has rank $i_{1}-1$. Let $G_{k} \in H^{0}\left(\mathbf{P}^{n}, \mathscr{O}_{\mathbf{p}^{n}}\left(d_{k}-d_{i_{0}}\right)\right)$ be chosen so that $G_{k}(x) \neq 0$ for $k=i_{1}, i_{1}+1, \ldots, i_{0}, \ldots, n-2$. Furthermore, there is some $m$ such that $z_{m}(x) \neq 0$. Hence

$$
\begin{gathered}
\left\{z_{m}\left(\frac{\partial F_{1}}{\partial z_{j}}, \ldots, \frac{\partial F_{n-2}}{\partial z_{j}}\right) \mid j=j_{1}, \ldots, j_{i_{1}-1}\right\} \\
\cup\left\{F_{i_{0}} G_{k} e_{k} \mid k=i_{1}, i_{1}+1, \ldots, n-2\right\}
\end{gathered}
$$

defines $n-2$ linearly independent vectors in $E_{x}$ when evaluated at $x$.

Thus, in any case, the evaluation map at $x$ is surjective and the claim follows.

In terms of the identifications in Proposition 2 and (2.6), (2.7) implies that the evaluation map

$$
T^{\prime} \otimes H^{0}\left(\mathbf{P}^{n}, \mathscr{O}_{\mathbf{P}^{n}}(\mu)\right) \rightarrow H^{0}\left(\mathbf{P}^{n}, E(\mu)\right)
$$

is not surjective. Therefore, by Proposition 1 , codim $T^{\prime} \geq \sum_{i=1}^{n-2} d_{i}-n$. But

$$
\operatorname{codim} T^{\prime} \leq \operatorname{codim}_{T_{S}\left(Y_{n}\right)} T_{S}(\pi(Z)) \leq \operatorname{codim}_{\widetilde{Y}_{n}} Z .
$$

Hence the theorem follows. 


\section{A NEW PROOF OF THE DENSITY THEOREM}

In this section, we denote $d=d_{1}, Y=Y_{3}$, and $N L_{d}=\Sigma_{3}$. Recall (cf. [3]) that the upper bound of the codimension of irreducible components of the Noether-Lefschetz locus $N L_{d}$ in the family $Y$ of smooth surfaces of degree $d$ in $\mathbf{P}^{3}$ is the geometric genus $p_{g}=\left(\begin{array}{c}d-1 \\ 3\end{array}\right)$ of any surface in $Y$. We will give a new proof of the following density theorem due to Ciliberto, Harris, and Miranda [5].

Theorem 2. For $d \geq 4$, the union of all irreducible components of $N L_{d}$ having codimension $p_{g}$ in $Y$ is dense in the classical topology.

Using an infinitesimal method, we will reduce the theorem to the following proposition.

Proposition 3. For each $d \geq 4$, there are some polynomials $G \in S^{2 d-4}$ and a surface $X \in Y$ with defining equation $F$ such that the map

$$
g: S^{d-4} \rightarrow S^{3 d-8} / J_{F, 3 d-8}
$$

defined by multiplication by $G$ is injective.

Proof. Let $F=z_{0}^{d}+z_{1}^{d}+z_{2}^{d}+z_{3}^{d}$ and

$$
G=\sum_{j=0}^{d-2} a_{j} z_{0}^{j} z_{1}^{j} z_{2}^{d-2-j} z_{3}^{d-2-j},
$$

where the constant coefficients $a_{j}$ 's are chosen so that every possible matrix of the form

$$
\left(\begin{array}{cccc}
a_{k} & a_{k+1} & \cdots & a_{k+m} \\
a_{k+1} & a_{k+2} & \cdots & a_{k+m+1} \\
\vdots & \vdots & \ddots & \vdots \\
a_{k+m} & a_{k+m+1} & \cdots & a_{k+2 m}
\end{array}\right)
$$

has nonzero determinant. Then we claim that $g$ is injective:

Without loss of generality, we may assume that a nonzero element of the kernel of $g$ is of the form

$$
P=\sum_{j=m_{1}}^{m_{2}} c_{j} z_{0}^{p+j} z_{1}^{q+j} z_{2}^{r-j} z_{3}^{s-j},
$$

where $p+q+r+s=d-4$ and $m_{1}<m_{2}$. This is because $G$ belongs to the span of the set of monomials $z_{0}^{i_{0}} z_{1}^{i_{1}} z_{2}^{i_{2}} z_{3}^{i_{3}}$ satisfying the equalities

$$
i_{0}=i_{1}=d-2-i_{2}=d-2-i_{3} .
$$

If we therefore break up $S^{d-4}$ into the span of monomials satisfying

$$
i_{0}-i_{1}=p-q, \quad i_{0}+i_{2}=p+r, \quad i_{0}+i_{3}=p+s,
$$


where $p, q, r, s$ vary but add up to $d-4$, and if we expand an element of ker $g$ in terms of these subspaces, then each piece also lies in ker $g$.

By symmetry of the role of $z_{0}$ and $z_{1}$, and of $z_{2}$ and $z_{3}$, we may assume $p \geq q$ and $r \leq s$. Then the limits of the sum above satisfy $m_{1} \geq-q$ and $m_{2} \leq r$. The condition that

$$
P \cdot G=\sum_{j, k} a_{j} c_{k} z_{0}^{j+k+p} z_{1}^{j+k+q} z_{2}^{d-2-j-k+r} z_{3}^{d-2-j-k+s} \in J_{F, 3 d-8}
$$

is equivalent to the system of equations

$$
\sum_{j+k=l} a_{j} c_{k}=0 \text { for } s \leq l \leq d-2-p .
$$

Since $m_{1} \geq-p$ and $m_{2} \leq s$, the two inequalities $m_{1} \leq k \leq m_{2}$ and $s \leq k+j \leq$ $d-2-p$ imply the inequality $0 \leq j \leq d-2$.

The coefficient matrix for the $c_{k}$ 's is

where

$$
A=\left(\begin{array}{cccc}
a_{\alpha} & a_{\alpha+1} & \cdots & a_{\beta} \\
a_{\alpha+1} & a_{\alpha+2} & \cdots & a_{\beta+1} \\
\vdots & \vdots & \ddots & \vdots \\
a_{\gamma} & a_{\gamma+1} & \cdots & a_{\delta}
\end{array}\right),
$$

$$
\alpha=s-m_{2}, \quad \beta=s-m_{1}, \quad \gamma=d-2-p-m_{2}, \quad \delta=d-2-p-m_{1} .
$$

The number of rows is

$$
\gamma-\alpha+1=d-2-p-s+1
$$

and the number of columns is

$$
\beta-\alpha+1=m_{2}-m_{1}+1 \text {. }
$$

But $(3.2)-(3.3)=d-2-p-s-\left(m_{2}-m_{1}\right) \geq d-2-p-s-(r+q)=2$. Therefore, $g$ is injective provided that the appropriate minors of the matrix of the $a_{j}$ 's of the form (3.1) are nonvanishing, and this may be arranged by taking the ratios $\left|a_{j+1} / a_{j}\right|$ to increase very rapidly with $j$.

Proof of Theorem 2. For a smooth surface $X \in Y, X \in N L_{d}$ if and only if $H_{\mathrm{pr}}^{1,1}(X) \cap H^{2}(X, \mathbf{Z}) \neq 0$. If there is a nonzero element $\gamma \in H_{\mathrm{pr}}^{1,1}(X) \cap$ $H^{2}(X, \mathbf{Q})$, then $m \cdot \gamma \in H_{\mathrm{pr}}^{1,1}(X) \cap H^{2}(X, \mathbf{Z})$ for some integer $m$ and hence $X \in N L_{d}$. For a given $\gamma \in H_{\mathrm{pr}}^{1,1}(X) \cap H^{2}(X, \mathbf{R})$, there are some elements of $H_{\mathrm{pr}}^{2}(X, \mathbf{Q})$ that are arbitrarily near to $\gamma$. We will show that one of these rational classes can be made to have type $(1,1)$ by making a small deformation of $X$.

We consider the universal family $\mathscr{F}$ of smooth surfaces of degree $d$ in $\mathbf{P}^{3}$ :

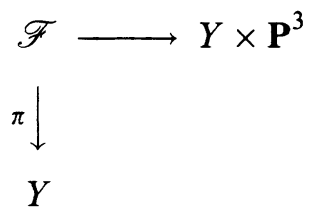


Since $\pi$ is a proper smooth map with maximal rank everywhere, Ehresmann's fibration theorem says that on a sufficiently small open neighborhood $U$ of $X$, there is a fiber preserving diffeomorphism

$$
\phi: \pi^{-1}(X) \times U \simeq \pi^{-1}(U)
$$

so that $\phi$ defines a diffeomorphism $\phi_{S}: X \rightarrow S$ and the induced map on the cohomology $\phi_{S}^{*}: H^{2}(S, \mathbf{C}) \rightarrow H^{2}(X, \mathbf{C})$ is an isomorphism for $S \in U$.

Let $R^{2} \pi_{*} \mathrm{C}$ be the second direct image sheaf of $\pi: \mathscr{F} \rightarrow Y$, which we recall is the sheaf associated to the presheaf

$$
U \rightarrow H^{2}\left(\pi^{-1}(U), \mathbf{C}\right),
$$

where $U$ runs through the open subsets of $Y$. Let $R_{\mathrm{pr}}^{2}$ be the kernel of a map

$$
L: R^{2} \pi_{*} \mathrm{C} \rightarrow R^{4} \pi_{*} \mathrm{C}
$$

defined as follows: For an open set $U \subset Y$ with $\pi^{-1}(U) \simeq \pi^{-1}(X) \times U$ as before,

$$
H^{2}\left(\pi^{-1}(U), \mathbf{C}\right) \simeq H^{2}(X, \mathbf{C}) .
$$

$L_{U}: R^{2} \pi_{*} \mathbf{C}(U) \rightarrow R^{4} \pi_{*} \mathbf{C}(U)$ is the cup product map with the associated $(1,1)$ form of $X$.

Then $R_{\mathrm{pr}}^{2}$ is a locally constant sheaf and there is a holomorphic vector bundle $\mathscr{H}$ on $Y$ associated to it, whose fiber over $S \in Y$ is $H_{\mathrm{pr}}^{2}(X, \mathbf{C})$. We have a Hodge filtration $F^{2} \subset F^{1} \subset F^{0}=\mathscr{H}$, and Hodge bundles $\mathscr{H}^{1,1}=F^{1} / F^{2}$ and $\mathscr{H}^{0,2}=F^{0} / F^{1}$, where the $F^{p}$,s are holomorphic vector bundles.

For a sufficiently small open neighborhood $U$ of $X$ as in (3.4), we can define a smooth map $f_{\mathbf{C}}$ on the total space of $\left.\mathscr{H}^{1,1}\right|_{U}$ as

$$
\begin{gathered}
f_{\mathbf{C}}:\left.\mathscr{H}^{1,1}\right|_{U}=\left\{(S, \gamma) \mid S \in U, \gamma \in H_{\mathrm{pr}}^{1,1}(S)\right\} \rightarrow H_{\mathrm{pr}}^{2}(X, \mathbf{C}), \\
(S, \gamma) \longmapsto \phi_{S}^{*}(\gamma) .
\end{gathered}
$$

Then $f_{\mathrm{C}}$ restricts to a map

$$
f: \mathscr{H}_{U, \mathbf{R}}^{1,1}=\left\{(S, \gamma) \mid S \in U, \gamma \in H_{\mathrm{pr}}^{1,1}(S) \cap H^{2}(S, \mathbf{R})\right\} \rightarrow H_{\mathrm{pr}}^{2}(X, \mathbf{R}) .
$$

We note that for the map $\pi_{1}: \mathscr{H}^{1,1} \rightarrow Y$, giving the bundle structure on $\mathscr{H}^{1,1}$,

$$
\pi_{1}\left(f^{-1}\left(H_{\mathrm{pr}}^{2}(X, \mathbf{Q})\right)\right)=N L_{d} \cap U .
$$

First, we will show that $f$ has maximal rank at some $\left(S_{0}, \gamma_{0}\right) \in \mathscr{H}_{U, \mathbf{R}}^{1,1}$. Then, by the Implicit Function Theorem, this implies that

$$
\begin{aligned}
& \text { there is an element } \gamma_{V} \in f(V) \cap H_{\mathrm{pr}}^{2}(X, \mathbf{Q}) \text { for each small open } \\
& \text { neighborhood } V \text { of }\left(S_{0}, \gamma_{0}\right) \text {, and } \operatorname{codim} \pi_{1}\left(f^{-1}\left(\gamma_{V}\right)\right)=p_{g} .
\end{aligned}
$$

In order to make the necessary computation, it is a good idea to distinguish the real tangent space $T_{S}(U)_{\mathbf{R}}$, the complexified tangent space $T_{S}(U)_{\mathbf{C}}$, and 
the holomorphic tangent space $T_{S}(U)$. There is of course a natural $\mathbf{R}$-linear isomorphism $T_{S}(U) \cong T_{S}(U)_{\mathbf{R}}$. Since $d f$ takes the tangent space of the fibers of $\pi_{1}$ to $H_{\mathrm{pr}}^{1,1}(X) \cap H^{2}(X, \mathbf{R})$, we obtain an induced $\mathbf{R}$-linear map

$$
\lambda: T_{S}(U)_{\mathbf{R}} \rightarrow \frac{H_{\mathrm{pr}}^{2}(X, \mathbf{R})}{H_{\mathrm{pr}}^{1,1}(X) \cap H^{2}(X, \mathbf{R})} \cong\left(H^{2,0}(X) \oplus H^{0,2}(X)\right) \cap H^{2}(X, \mathbf{R})
$$

having maximal rank if and only if $f$ does. Under the R-linear identifications $T_{S}(U)_{\mathbf{R}} \cong T_{S}(U)$ and

$$
\left(H^{2,0}(X) \oplus H^{0,2}(X)\right) \cap H^{2}(X, \mathbf{R}) \cong H^{0,2}(X),
$$

the map $\lambda$ is identified with the derivative of the period map

$$
T_{S}(U) \rightarrow H^{0,2}(X) .
$$

By the work of Griffiths [14], the derivative of the period map is the composition of the Kodaira-Spencer map $\rho$ with the cup product with $\gamma$, i.e.

$$
T_{S}(Y) \stackrel{\rho}{\longrightarrow} H^{1}\left(S, \Theta_{S}\right) \stackrel{\cup \gamma}{\longrightarrow} H^{0,2}(S) .
$$

Thus $\lambda$, and hence $f$, has maximal rank if and only if

$$
\cup \gamma: \rho\left(T_{S}(Y)\right) \rightarrow H^{0,2}(S)
$$

is surjective, or equivalently,

$$
H^{2,0}(S) \stackrel{\cup \gamma}{\longrightarrow} \rho\left(T_{S}(Y)\right)^{*} \text { is injective. }
$$

Referring to (2.6),

$$
\rho\left(T_{S}(Y)\right) \simeq S^{d} / J_{F, d},
$$

where $F$ is the defining equation of $S$. By Macaulay's theorem (see, e.g., [9, Theorem 2.15]),

$$
\left(\rho\left(T_{S}(Y)\right)\right)^{*} \simeq S^{3 d-8} / J_{F, 3 d-8} .
$$

In terms of the identifications in (2.1) and above, the above map (3.6) is injective if the multiplication map

$$
g: S^{d-4} \rightarrow S^{3 d-8} / J_{F, 3 d-8}
$$

is injective, where $g$ is the multiplication by $G(\gamma) \in S^{2 d-4}$ corresponding to $\gamma$. By Proposition $3, g$ is injective at $\left(S_{0}, \gamma_{0}\right)$, where

$$
S_{0}=\left\{z_{0}^{d}+z_{1}^{d}+z_{2}^{d}+z_{3}^{d}=0\right\}
$$

and $\gamma_{0}$ corresponds to $G \in S^{2 d-4} / J_{F, 2 d-4}$ with some fixed real coefficients $a_{i}$ 's, and hence $\gamma_{0} \in H_{\mathrm{pr}}^{1,1}\left(S_{0}\right) \cap H^{2}\left(S_{0}, \mathbf{R}\right)$. So $f$ has maximal rank $p_{g}$ at $\left(S_{0}, \gamma_{0}\right)$. 
In fact, $\rho$ composed with the cup product map $\cup \gamma$ gives rise to a holomorphic map of vector bundles on $\mathscr{H}^{1,1}$ so that we can define a map

$$
\begin{aligned}
\sigma: \mathscr{H}^{1,1} & \rightarrow \Theta_{Y}^{*} \otimes \mathscr{H}^{0,2}, \\
(S, \gamma) & \mapsto \sigma(S, \gamma): \Theta_{Y} \rightarrow \mathscr{H}^{0,2} .
\end{aligned}
$$

The locus $A$ where $\sigma(S, \gamma)$ drops rank is an analytic subvariety of $\mathscr{H}^{1,1}$. Since $\sigma\left(S_{0}, \gamma_{0}\right)$ has maximal rank $p_{g}, A$ is proper. Since $f$ has maximal rank at $\left(S_{0}, \gamma_{0}\right), A \cap \mathscr{H}_{\mathbf{R}}^{1,1}$ is also proper, where $\mathscr{H}_{\mathbf{R}}^{1,1}=\left\{(S, \gamma) \mid \gamma \in H_{\mathrm{pr}}^{1,1}(S) \cap\right.$ $\left.H^{2}(S, \mathbf{R})\right\}$. Hence, (3.5) holds for every $(S, \gamma) \in \mathscr{H}^{1,1}$ and the theorem follows.

\section{REFERENCES}

1. E. Arbarello, M. Cornalba, P. Griffiths, and J. Harris, Geometry of algebraic curves. I, Springer-Verlag, Berlin, 1985.

2. R. Bott, Homogeneous vector bundles, Ann. of Math. (2) 66 (1957), 203-248.

3. J. Carlson, M. Green, P. Griffiths, and J. Harris, Infinitesimal variations of Hodge structure. I, Compositio Math. 50 (1983), 109-205.

4. J. Carlson and P. Griffiths, Infinitesimal variation of Hodge structure and the global Torelli problem, J. Géométrie Algébrique d'Angers, Sijthoff and Noordhoff, 1980, pp. 51-76.

5. C. Ciliberto, J. Harris, and R. Miranda, General components of the Noether-Lefschetz locus and their density in the space of all surfaces, Preprint, 1988.

6. P. Deligne, Theorie de Hodge. II, Inst. Hautes Études Sci. Publ. Math. 40 (1971), 5-58.

7. L. Ein, An analogue of Max Noether's Theorem, Duke Math. J. 52 (1985), 689-706.

8. M. Green, Koszul cohomology and the geometry of projective varieties. II, J. Differential Geom. 20 (1984), 279-289.

9. _ـ The period map for hypersurface sections of high degree of an arbitrary variety, Compositio Math. 55 (1984), 135-156.

10. (1988), 155-159.

11. _ Components of maximal dimension in the Noether-Lefschetz locus, J. Differential Geom. 29 (1989), 295-302.

12. __ Griffith's' infinitesimal invariant and the Abel-Jacobi map, Preprint.

13. __ Koszul cohomology and geometry, Preprint.

14. P. Griffiths, Periods of integrals on algebraic manifolds. I, II, Amer. J. Math. 90 (1968), 568-626, 805-865.

15.

16. $\ldots$, Periods of integrals on algebraic manifolds. III, Inst. Hautes Études Sci. Publ. Math. 63 (1970), 125-180.

17. P. Griffiths and J. Harris, On the Noether-Lefschetz Theorem and some remarks on codimension-two cycles, Math. Ann. 271 (1985), 31-51.

18. _ Principles of algebraic geometry, Wiley-Interscience, New York, 1978.

19. R. Hartshorne, Algebraic geometry, Springer-Verlag, New York, 1977.

20. R. Lazarsfeld, A sharp Castelnuovo bound for smooth surfaces, Duke Math. J. 55 (1986), 423-429.

21. S. Lefschetz, On certain numerical invariants of algebraic varieties, Trans. Amer. Math. Soc. 22 (1921), 326-363. 
22. __ L'analysis situs et la geometrie algebrique, Gauthier-Villars, Paris, 1924.

23. A. Lopez, On the Picard group of projective surfaces, Thesis, Brown Univ., Providence, R.I., 1988.

24. D. Mumford, Lectures on curves on an algebraic surface, Ann. of Math. Studies, no. 59, Princeton Univ. Press, Princeton, N.J., 1966.

25. M. Noether, Zur Grundlegung der Theorie der algebraischen Raumcurven, Berliner Abh., Berlin, 1882.

26. C. Voisin, Une precision du théorème de Noether, Preprint.

27. __ Composantes de petite codimension du lieu de Noether-Lefschetz, Preprint.

Department of Mathematics, University of California, Los Angeles, California 90024

Current address: 56 Stephen Hopkins Court, Providence, R.I. 02904 